\title{
Segmentation of retinal blood vessels for detection of diabetic retinopathy: A review
}

\author{
Rezty Amalia Aras*, Tri Lestari, Hanung Adi Nugroho, Igi Ardiyanto \\ Department of Electrical Engineering and Information Technology, Universitas Gadjah Mada \\ Jalan Grafika No.2 BulakSumur, Yogyakarta 55281, Indonesia
}

Article history:

Received: 25 May 2016/ Received in revised form: 29 May 2016/ Accepted: 29 May 2016

\begin{abstract}
Diabetic detinopathy (DR) is effect of diabetes mellitus to the human vision that is the major cause of blindness. Early diagnosis of DR is an important requirement in diabetes treatment. Retinal fundus image is commonly used to observe the diabetic retinopathy symptoms. It can present retinal features such as blood vessel and also capture the pathologies which may lead to DR. Blood vessel is one of retinal features which can show the retina pathologies. It can be extracted from retinal image by image processing with following stages: preprocessing, segmentation, and post-processing. This paper contains a review of public retinal image dataset and several methods from various conducted researches. All discussed methods are applicable to each researcher cases. There is no further analysis to conclude the best method which can be used for general cases. However, we suggest morphological and multiscale method that gives the best accuracy in segmentation.
\end{abstract}

Keywords: Diabetic Retinopathy; Digital Image Processing; Segmentation Methods; Retinal Blood Vessel

\section{Introduction}

The term diabetes mellitus (DM) is used to describe metabolic disorders from several etiologies of metabolism process such as carbohydrate, fat, and protein resulting from defects in insulin disorder [1]. According to Global Prevalence of Diabetes, in the year 2000, it was estimated that there were 171 million of diabetes patient in the whole world and it will be doubled to 366 million by 2030[2]. While in Indonesia, World Health Organization (WHO) predicted that people with diabetes will rise from 8.4 million in 2000 to 21.3 million in 2030 , or the $4^{\text {th }}$ country with the highest number of people with diabetes in the world [3].

One of the diseases caused by the diabetes is retinal disease called diabetic retinopathy (DR). Diabetic retinopathy is a very specific retinal vascular complication of diabetes mellitus. It is the main cause of sight loss on people of working age in United States of America. Early diagnosis for diabetes and diabetic retinopathy is necessary for reducing the potential of sight loss [4].

Analysis and detection structural changes on the retinal blood vessel of fundus image is very important to diagnose and detect retinal disease such as diabetic retinopathy, hypertension, age-related macular degeneration (AMD), and arteriosclerosis [5]. Early symptoms of those diseases are indicated by retinal blood vessel's features such as diameter, length, angle, and tortuosity [6]. Retinal blood vessel is the

* Corresponding author. Tel: +62-852-998-92667; fax: +62-852-998-92667 Email: rezty.mti15@mail.ugm.ac.id. only part of the blood circulation that could be directly observed and studied in detail. Retinal imaging is further used to determine retinal normality and to diagnose or monitor retinal abnormalities. Changes in width and tortuosity of the retinal blood vessel are believed as indicators for diabetic retinopathy risk level [7].

Various researches regarding to blood vessel detection using advanced methods have been conducted by researchers in the last few years. From those researches, it may be concluded that blood vessel detection for diabetic retinopathy diagnosis in digital fundus image basically consists of three steps procedures; pre-processing, segmentation, and postprocessing.

Many paper are proposed for blood vessel segmentation of retinal vessel to diagnosis of Diabetic Retinopathy such as "Review of retinal blood vessel detection methods for automated diagnosis of Diabetic retinopathy" by Preethy et al. [8], "Blood vessel segmentation methodologies in retinal images - A survey" by Owen et al. [9], and "A survey on Blood Vessel Segmentation Methods in Retinal image" by Navdeep et al. [10]. This paper is compiled in the following arrangement. In the first section contains elaboration of blood vessel detection on diabetic retinopathy. The second section explains the used database of retinal fundus image. The third section explains about pre-processing method. The fourth section explains about segmentation method of retinal blood vessel. The fifth section explain about post-processing method. Afterwards, the sixth section followed by discussion. Finally, the last section will draw the conclusion. 


\section{Retinal Image Database}

The first step in image analysis process is to determine the retinal image to be analyzed. Retinal image may be collected from direct image shooting or from publicly available database. Some database which are available for retinal image processing, are as follows:

\subsection{DRIVE Database}

DRIVE (Digital Retinal Image for Vessel Extraction) is a fundus image database which available for public. Fundus images in this database were collected through diabetic retinopathy screening program in Netherland, from 453 people in the age of 31-86 years old. Fundus images in this database sums up to 40 images with 20 training and 20 testing images [11].

\subsection{STARE Database}

STARE Database contains 20 images for blood vessel segmentation. Image size on this database is $700 \times 605$ pixels with 8 bit per color channel [12].

\subsection{ARIA Online}

This database was recorded in 2006 in research collaboration between St. Paul's Eye Unit, Royal Liverpool University Hospital Trust, Liverpool, UK, and the Department of Ophthalmology, Clinical Sciences, University of Liverpool, Liverpool, UK. The database consists of three groups: 92 images of age based macular degeneration group, 59 images of retinas that are infected with diabetes group, and 61 images of control group. Standard references used in this database are blood vessel, optical disc, and location of fovea. Images on this database are RGB images with $768 \times 576$ pixels with TIFF format [9].

\subsection{Image Ret}

This database was recorded in 2008 and divided into two sub databases namely, DIARETDB0 and DIARETDB1. DIARETDB0 contains 130 retina images, 20 of them are normal images while the other 110 are images with various symptoms of diabetic retinopathy. Meanwhile, DIARETDB1 consists of 89 retina images with 5 healthy images and 84 images with light symptoms of diabetic retinopathy. These sub databases were determined by diabetic retinopathy symptoms such as microaneurysms, hemorrhages, hard exudates, and soft exudates. Image size on the database is $1500 \times 1152$ in PNG format [9].

\subsection{Messidor}

Messidor project database is the largest database with 1200 retina images. These images were collected from 3 different ophthalmology departments with the resolutions of 1440x960, $2240 \times 1488$, and $2304 \times 1536$ pixels. Images in this database were saved in TIFF format. Out of 1200 images, there are 800 images with pupil dilation [13].

\subsection{Review Database}

This database contains retinal blood vessel images which used to estimate blood vessel's width. This database was made by Department of Computing and Informatics, University of Lincoln, Lincoln, UK in 2008 [14].

\subsection{ROC Microaneurysm Set}

This database consists of 100 colored fundus images with microaneurysm. The images in this database are $768 \times 576$, 1058x1061, and 1389x1383 pixels in JPEG format [9].

\subsection{VICAVR Database}

VICAVR database is a series of retina images used for AVR or Artery to Vena ratio. This database consists of 58 images with $768 \times 584$ pixels resolution [15].

\subsection{CHASE_DBI Database}

CHASE_DBI database is a dataset consist of 28 colored retinal fundus images from Child Heart and Health Study Program patients in England [16].

\subsection{Retinal Vessel Tortuosity Dataset}

Retinal vessel tortuosity (RET-TORT) dataset consist of normal and hypertensive patient's retinal image and the information about manually estimated tortuosity. Total of the images are 60 retinal vessel image [17].

\section{Pre-processing Method}

Pre-processing is used to remove the noise on retina images and to increase the image contrast value on fundus images. Moreover, pre-processing is also used for image normalization and brightness correction to increase the accuracy for the next step.

\subsection{Brightness Level Equalization}

\subsubsection{Green Channel}

Retina's features or traits can be differentiated by using color as feature descriptor. Saleh et al. [18] identified that green channel generates maximum contrast on retina image, thus differentiating one feature to the other. In reserach of Patwari et al. [19] green channel shows higher contrast intensity compared with red and blue channel, while Elbalaoui et al. [20] said that green channel has the best contrast compared with the red and blue channels.

\subsubsection{Image Normalization}

Image normalization is used to minimize intra-image variable in fundus images. Kumar et al, stated that color normalization could be utilized for brightness correction, color modification, and contrast enhancement [21]. Fraz et al, in his research found a variation on intensity of the macula 
background to the surrounding area that may affect the segmentation process of the blood vessel. To minimize this variation, the reduction of background estimation from the original image is implemented by using $31 \times 31$ arithmetic mean kernel [22]. It was similar with $\mathrm{L}$. $\mathrm{Xu}$ et al. who reduced background estimation from the original image using $25 \times 25$ median filter for background normalization [23]. Foracchia et al. made parameter model to estimate lighting and variable contrast for normalization. This method claimed that it could significantly reduce the image variation [24]. Vlachos et al. used this model for background normalization [25]. Goatman et al. proposed a method for contrast enhancement using image contrast to cover the whole pixel coverages for normalization [26]

\subsubsection{Hue Channel}

Hue channel in pre-processing plays a role in increasing the blood vessel's contrast to the background by mapping the original intensity value between 0 and 1[11] [15].

\subsubsection{Matched Filtering}

Gaussian Shaped is method of matched filter to enhance the blood vessel from the retinal vessel cross section of retinal image. Thin vessel is the main problem of vessel enhancement process. Q. Li et al. [27] proposed a multiscale matched filter technique to solve this problem. The proposed method could detect both large and small vessels. This method delivers an efficient process to remove noise, so thin vessels can be identified with a precise width evaluation.

\subsubsection{Homomorphic Filtering}

Homomorphic filtering is a common technique for image enhancement and correction for normalizes the brightness of the whole image and increase the contrast since illumination and reflectance are the component that could not be separated. Two component should be used to improve the image appearance simultaneously, which is the brightness and contrast [28]

\subsubsection{High Pass Filter}

High pass filter is a technique that makes an image sharper and make the details of image clearer [28]. This method performs same process as low-pass filter with a different convolutional kernel.

\subsection{Contrast Enhancement}

\subsubsection{Morphological Operation}

Akram et al. [29] proposed a morphological operation to soften the optic disc and brighten the lesions. Marwan et al. [30] used top-hat and bottom-hat transform for contrast enhancement and exhibited dark lesions with minimum background variations. Sun et al. proposed a multiscale morphological operation for blood vessel's enhancement [31]. Afterwards, Giancardo et al. proposed a morphological reconstruction for vessel enhancement, such as vessel structure [32].

\subsubsection{Gabor Wavelet}

Gabor wavelet is used to enhance thin and barely seen vessel pattern. However, the result of Gabor wavelet have a slight blur effect, so non-sharp filter is used to sharpen the vessel's edge in order to make the vessel clearer than its original image [33]

\subsubsection{Histogram Equalization}

Histogram equalization aims to distribute intensity value an image input to produce output with even intensity. Tamilarasi et al. used histogram equalization combined with smoothing filter to enhance retina image's contrast [34]. Antal et al. use histogram equalization (CLAHE) that applied local histogram to deferent area of the input image[35]. in CLAHE, contrast limitation is applied to every pixel to avoid noise amplication. Elbalaoui et al. [20] used CLAHE because it can increase the contrast between contours. When enhancing the contrast image, two factors must be considered, spped and efficiency. Bhattacharjee et al. using CLAHE to enhance the contrast of fundus images which operates on a small region of retinal images, but can generate noise in image [36]. Other contrast enhancement method that can be done with adaptive histogram equalization is by contrast limited adaptive histogram [37]. In this method, the image is split to 8x8 sections and histogram equalization is applied locally to every section of the image [38]. This method is very effective in emphasizing local values in an image. However, this method also generates noise that can disturb the next process [39]. Other histogram equalization development is illumination equalization which uses global local adaptive histogram equalization with partially overlapped windows (GLAPOW) algorithm [40]. This technique has a good performance compared with other technique for image fundus. Other contrast enhancement method that also adopts histogram technique is gray level grouping [41]. This method has similarities with histogram equalization technique, but differs on gray level grouping aspects based on its frequency of occurrences.

\section{Segmentation Method of Retinal Blood Vessel}

Segmentation process is the core process in blood vessel detection on retinal fundus image. In this process, blood vessel will be detected on eye retinal fundus image. Detected blood vessel in this segmentation process can be used for further research. The segmentation method covered in this paper is divided in to three major groups namely; image based, pattern recognition based, and vessel tracking/tracing.

\subsection{Image Based}

Segmentation methods categorized in this group are based on digital image processing operations. These techniques were using image as the primary data. It differentiates these techniques from pattern recognizing technique which requires input image transformation process into values or features. This group may be divided into two major divisions, namely, edge/boundary based and region based or region growing.

\subsubsection{Edge/Boundary Based}

4.1.1.1. Canny Edge Detection

Canny edge detection is used to detect edges [42] Chhabra 
et al. [11] applied canny edge detection method for each intensity profile to find the edge of the vessel. The weakness of this method is the lack of segmented image and feature to be extracted.

\subsubsection{Multiscale}

Multiscale is a method for blood vessel segmentation in eye retinal fundus image. Nguyen et al. [14] used multiscale line detector method for blood vessel segmentation and Moghimirad et al. [43] proposed multiscale method for retinal blood vessel segmentation based on weighted twodimensional (2D) medialness function.

Frangi et al. studied multiscale second order local structure of images (hessian) in the context of vessel filter's enhancement [44]. Vessel dimensions were derived through Eigen value analysis of the hessian where the second order structure of the image can be outlined. This may directly give the smallest curvature direction along the vessel. Vessel's dimension is defined using geometric ratio, Eigen value, and Frobenius norm matrix.

Martinez-Perez et al. provided scale space analysis method, based on blood vessel's width, size, and orientation [45]. This method uses two main geometrical features and the second order of the intensity (edge and maximum curvature) along the scale space that gives information regarding the image.

Vlacos et al. [46] proposed multi sale line tracking for blood vessel segmentation. Line tracking is initialized in various scales to accommodate various vesselwidths.

Tsai et al. [47] proposed automatic vessel segmentation method using hessian matrix. They first choose 25 frames from the contrast's angiogram to obtain the vessel structure. They subsequently define adaptive feature transformation equations using gray values and scale to improve feature response.

Han et al. [48] designed multiscale local statistical features basen on gray level and morphological fatures combined with features based local area shape. This method has great visual presence, which good for describe shape and structure of retinal blood vesselimage.

\subsubsection{Matched Filter Method}

Matched filter is template based method that uses two dimensional linier structure element with Gaussian crossprofile section that is changed or rotated into three dimension to identify the blood vessels cross profile. The result of this process is a binary segmentation of the blood vessel. This method's weakness lies in the image detection, small vessels are not defined and cannot be validated.

Chaudhuri et al. [49] Proposed two dimensional linear kernel with Gaussian profile for retinal blood vessel segmentation. Profile from the filter is designed to be matched with the blood vessel. Kernel is gradually rotated 15 degrees to fit in different vessels. The highest response of the filter is selected for every pixel and thresholding to generate binary image. Next process is to remove other features besides the vessel. Hoover et al. combined local traits and retinal blood vessel area for segmentation using threshold probing technique on image matched filter [50]. To increase the threshold, this method using iterative of MFR image area. Image analysis method MFR was formulated by Chaudhuri et al [49]. Thresholding with iterative probing is conducted on every part for every pixel as vessel or non-vessel. Al-Rawi et al. [51] developed Chaudhuri's et al. matched filter method using search optimization procedure to find the best parameter for matched filter size, deviation standard, and threshold value.

The other matched filter technique is proposed by Cinsdikici and Aydin that developed a hybrid method from matched filter and Ant Colony Algorithm for retinal blood vessel segmentation [52]. Their experiments show the accuracy improvement of the matched filter. The next generation of matched filter method is developed by Odstrcilik et al. [53] and Fazli et al. [54]. Odstrcilik et al. [53] made automatic retinal blood vessel segmentation method. This concept develops two dimensional matched filter. It uses the correlation between local image area and 2D masks matched with vessels width. Fazli et al. [54] uses Gaussian matched filter and its first derivative for retinal blood vessel segmentation. Xiang et al. improved Odstrcilik method using 2D Gaussian matched filter for retinal blood vessel enhancement and simplified with Neural Network. Next 2D Otsu algorithm is used to find the best segmentation result and the vessel generated through regional connectivity analys is [55].

The new matched filter technique proposed by Kawadiwale et al. [56] that conducted retinal blood vessel segmentation using matched filter with two different values from varians parameter. The used filtering technique is classical matched filtering, proposed modified matched filtering, second derivative from Gaussian function filtering, K-means, and Fuzzy C-means clustering.

\subsubsection{Gabor Filter}

Gabor filter is widely used for image processing application including image segmentation and image analysis [27] Farokhian et al. [57] research applied Gabor filtering technique to detect blood vessels in retina image based on wavelet gabor because gabor filter are better for the segmentation. It can detect specific features in retinal image.

\subsubsection{Region Based/Region Growing}

The vessels segmentation process can also be prefixed with region growing technique to begin the process of reconstruction of blood vessels in retina of fundus image.

Moghimirad et al. [43] developed vessel reconstruction with vessels radius estimation based on information obtained from vessel centerline extraction use eigenvalue and result of medialness function and vessel reconstruction using the estimated radius and the vessel's graph. while on research of Qian Zhao et al. [12] applied region growing for vessel segmentation thin curved to solve the failing segmentation for retinal vessels by previous level set method. Pattern recognition based

\subsubsection{Feature Extraction}

Segmentation process with classification technique requires the features extraction. It is necessary for performing an efficient segmentation process. Not all pixels in the image are segmented but only certain features in an image. The process of defining the features or characteristics of the image 
that most efficient or more meaningful, which represents important information for analysis and classification. This stage extracts features of pixels in image [58].

\subsubsection{Morphological Method}

Morphological method is used to extract image component and determine the region such as, feature, boundaries, and skeleton. Two main morphological operators which used are dilation and erosion. Dilation expands the object with structuring the element, filling the holes, and connecting the area disjoint, while erosion is depreciation object to the structuring element. The other morphological operation is closing and opening. Opening is erosion followed by dilation, while closing is dilation followed by erosion.

Zana et al. combine morphological filter with cross curvature evaluation to segment vessel pattern [59]. The result of this method is relatively strong. Afterward, they merge morphological multiscale enhancement by using fuzzy filter and watershed transformation to extract vascular tree in angiogram, using the method of Sun et al. [60]. This method gets important information from shape and area of the vascular. And then, Fraz et al. proposed vessel centerline detection combination and morphological bit plane slicing to extract blood vessel of retinal image [61]. This method works perfectly in feature of vessel with Gaussian Shaped Profile, but it less suitable for retinal image where arterioles are clear. Other method to improved quality of retinal image is declared by Singh et al. [62] which proposed an approach to detect blood vessel with use the morphological to eliminate background and enhancement blood vessel with phase preserving noise removal algorithm. Vessel silhouette is extracted with the fixed threshold scheme. Sindhu et al. segmented blood vessel by using morphological operations with applied threshold [63]. This method is fast and efficient and can be applied to a large amount of images for automatic segmentation.

\subsubsection{Pattern Classification and Machine Learning}

Pattern classification in blood vessel segmentation is divided into two categories, supervised method and unsupervised method. Supervised method uses label information before deciding whether the pixel is vessel or not, while unsupervised method segments vessel without previous labeling [9].

\subsubsection{Convolutional Neural Network}

Convolutional Neural Network $(\mathrm{CNN})$ is used as hierarchical features extraction, e.g. Wang et al. [64] Each targeted pixel, the raw pixel values from the rectangular window is centered and directly given as $\mathrm{CNN}$ input. These methods usually fail to capture some of good vessels, but actually these vessel are not essential to the clinical importance.

\subsubsection{Classification}

\subsubsection{K-Nearest Neighbor (KNN)}

KNN classifier operates on the premise that classification of unknown object can be done by relating it to the known according some distance or similarity function. Saranya et al. [65] classifying blood vessel using $\mathrm{K}$ nearest neighbor (KNN) classifier. This method has good performing in segmenting small blood vessel.

\subsubsection{Neural Network}

Neural network is one of the popular classification method in biomedical research. Chhabra et al. [11] proposed a neural network classifier for classifying the pixel of vessel into artery and vein. This method gives good performance in classify artery and vein but its performance may be different in other cases. In the other research, Wilfred et al. [66] used backpropagation neural network to classify pixel of retinal image into vessel and non-vessel. It ensures the best performance in the classification stage.

\subsubsection{Thresholding}

Fraz et al. [17] proposed thresholding method to classify pixel into vessel and non-vessel. This method can classify image into separate component which changes the image into a binary image. The above method uses gray values for performing the pixel classification. Thresholding process is used for removing unimportant details. Quality of segmentation can be shown from select threshold value.

\subsubsection{Clustering}

Saranya et al. [65] proposed segmentation method using fuzzy C-means (FCM). FCM is an overlapping clustering algorithm, where each point in image may belong to two or more cluster with different degree of membership. Features with high similarity in the image are grouped into the same cluster. The similarity is defined by the distance of the feature vector to cluster centers. Fuzzy partitioning is carried out through an iterative optimization of the object fuction.

\subsection{Vessel Tracing/Tracking}

Vessel tracking method separates vessel between two points using local transformation and works only in one point, not all of the points. Vessel tracking is one of segmentation algorithm which is frequently used. Basically, vessel tracking is principal curve projection algorithm, e.g. research conducted by You et al. [37] which used vessel tracking method in segmenting vessel into three attributes of direction, width, and center point. The density distribution of the cross section of a blood vessel is estimated by using a Gaussian shaped function. Individual segments are identified by using a search procedure, which kept track of the center of the vessel and makes some decisions about the future path of the vessel based on certain vessel properties. However, the vesseltracking method requires a user intervention and may be confused by vessel crossing and bifurcations. To deal with the problem of the central light reflex area in the tracking method, the vessel intensity profiles can be modeled as twin Gaussian functions, and a new method in which the tracking process started from the circumference of the optic disc and applied a Kalman filter as the base to estimate the next search location. It was developed by Chutatape et al. [67] that proposed tracking method with Gaussian and Kalman filter to detection retinal vessel in retinal images. Gaussian matched filter used for estimating the center point of vessel and Kalman filter used for estimating location next vessel segment. Thus, 
Delibasis et al. [68] proposed automatic tracing model to segment and estimate the vessel. The algorithm is used vessel parametric model. This model describes line as definition of parameter. MoM (measure of match) is used to calculate the similarity between model and image. Pixel initialization for tracking vessel is realized by using multiscale vesselness filter and divides the binary output and chooses which pixel with null value as seed. Vessel tracking is obtained from the best stripe identification which is matched with the vessel by using the seed point, strip orientation, size of stripe and MoM. The new research of tracking method is tracing of retinal blood vessel through edge information by Khadijah et al. [69] that improved algorithm for tracking vessel. This method can be the basic for an automated detection disease such as stroke and diabetic.

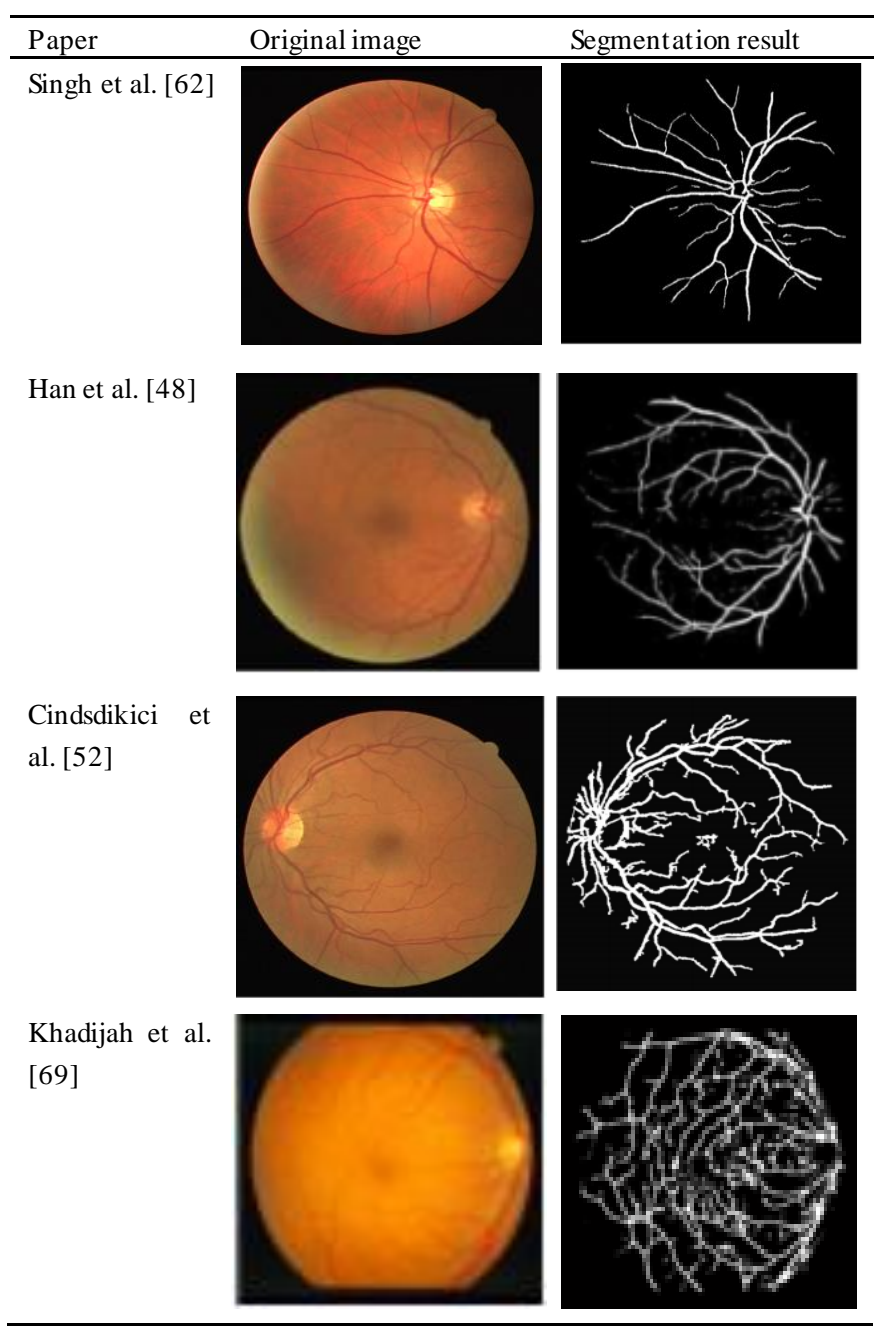

Fig. 1. Comparison bet ween originalimage and segemnation result of retinal fundus image

\section{Post-processing Method}

Post-processing is the final process in the research. This process aims to increase, sharpen, and correct the segmentation process results. Post-processing makes a better result of detection and segmentation. The post-processing can also remove unnecessary region and focus to the vessel [62]

Some post-processing techniques are implemented to increase the accuracy of segmentation [27]. The segmented image then convolved with $3 \times 3$ median filter to reduce salt and pepper noise. Length filtering along with some morphological operators are used to remove isolated pixel [70] and Qian zhao et al. [12] proposed two steps of postprocessing from the final segmentation result. The first step is combined with different retinal vessel segmentation results which derived from the level set method and region growing. The second step is to remove false detection of the isolated vesselpixels.

\section{Discussion}

In this review, we discuss about segmentation techniques, which start from image acquisition of public retinal fundus image database, pre-processing, segmentation, and postprocesing. See table 1. For Some of segmentation method of retinal blood vessel implementation and it's accuracy on retinal fundus image database. Based on segmentation method that described in this review, the highest accuracy method result is improved morphological method by Singh et al. [62] and improved multiscale method by Han et al. [48]. These methods compared DRIVE and STARE database. Singh et al. proposed a new morphological method based approach for blood vessel segmentation in retinal image. It used to remove background and enhance blood vessel, the result of this method work accurately and efficiently in image database [62]. Han et al. developed multiscale local statistical features based on gray level and morphological fatures combined with features based local area shape. This method has great visual presence, which good for describe shape and structure of retinal blood vessel image. The problem of this method is long time for training dan not detect thin vessel which caused low contrast image [48]. The future work to get higher performance of segmentation is to develop algorithm for removing optic disk and classify vessel into arteries and veins to detect diabetic retinopathy and to resolve the thin vessel problem can be done by combine with other method that can present thin vessel.

\section{Conclusion}

This paper evaluates methods for detection of diabetic retinopathy through vessel segmentation of retinal image. The processes involve image database, pre-processing, segmentation and post-processing. All discussed methods are applicable to each researcher's unique cases. There is no further analysis to conclude the best method in general. Each method has advantages and disadvantages, which depends on the condition and needs of researchers. However, from each method in this review we suggest to use morphological method by Singh et al. [62] and multiscale method by Han et al. [48] because the method show the good accuracy in segmentation which use STARE and DRIVE database. 
Tabel 1. Performance measure of segmentation method

\begin{tabular}{|c|c|c|c|c|c|c|}
\hline \multicolumn{7}{|c|}{ Morphological Method } \\
\hline Paper & Year & Title & Database & Sensitivity & Specificity & Accuracy \\
\hline Zana et al. [59] & 1999 & $\begin{array}{c}\text { A mult timodal registration algorithm of eye fundus } \\
\text { images using vessels detection and hough } \\
\text { transform }\end{array}$ & DRIVE & 0.6971 & - & 0.9377 \\
\hline Fraz et al. [61] & 2011 & An approach to localize the retinal blood vessels & DRIVE & 0.7152 & 0.9769 & 0.9452 \\
\hline & & using bit planes and centerline detection & ST ARE & 0.7311 & 0.9680 & 0.9440 \\
\hline Singh et al. [62] & 2014 & A new morphology based approach for blood & DRIVE & - & - & 0.9460 \\
\hline & & vessel segmentation in retinal images & ST ARE & - & - & 0.9521 \\
\hline Sindhu et al. [63] & 2013 & $\begin{array}{c}\text { Aut omated retinal vessel segmentation using } \\
\text { morphological operation and threshold }\end{array}$ & DRIVE & 0.96 & 0.89 & 0.93 \\
\hline \multicolumn{7}{|c|}{ "Matched Filter Me thod } \\
\hline Paper & Year & Title & Database & Sensitivity & Specificity & Accuracy \\
\hline Chaudhuri et al. & 1989 & Detection of blood vessels in retinal images using & DRIVE & - & - & 0.8773 \\
\hline [49] & & two dimensional matched filters & ST ARE & - & - & \\
\hline Hoover et al. [50] & 2000 & $\begin{array}{l}\text { Locating blood vessels in retinal images by } \\
\text { piecewise threshold probing of a matched filter } \\
\text { response }\end{array}$ & ST ARE & 0.6751 & 0.9567 & 0.9267 \\
\hline $\begin{array}{l}\text { Al-Rawi et al. } \\
{[51]}\end{array}$ & 2007 & $\begin{array}{l}\text { An improved matched filter for blood vessel } \\
\text { detection of digital retinal images }\end{array}$ & DRIVE & - & - & 0.9535 \\
\hline $\begin{array}{l}\text { Cinsdikici and } \\
\text { Aydin [52] }\end{array}$ & 2009 & $\begin{array}{l}\text { Detection of blood vessels in ophthalmoscope } \\
\text { images using MF/ant (matched filter/ant colony) } \\
\text { algorithm }\end{array}$ & DRIVE & - & - & 0.9293 \\
\hline $\begin{array}{l}\text { Odstrcilik et al. } \\
\text { [53] }\end{array}$ & 2013 & $\begin{array}{l}\text { Retinal vessel segmentation by improved matched } \\
\text { filtering: evaluation on a new high-resolution } \\
\text { fundus image database }\end{array}$ & $\begin{array}{l}\text { DRIVE } \\
\text { ST ARE }\end{array}$ & $\begin{array}{l}0.7060 \\
0.7847\end{array}$ & $\begin{array}{l}0.9693 \\
0.9512\end{array}$ & $\begin{array}{l}0.9340 \\
0.9341\end{array}$ \\
\hline Fazli et al. [54] & 2013 & $\begin{array}{l}\text { A Novel Retinal Vessel Segmentation Based On } \\
\text { Local Adaptive Hist ogram Equalization }\end{array}$ & DRIVE & - & - & 0.9353 \\
\hline \multicolumn{7}{|c|}{ "Vessel Tracking/ Tracing Method } \\
\hline Paper & Year & Title & Database & Sensitivity & Specificity & Accuracy \\
\hline $\begin{array}{c}\text { Khadijah et al. } \\
\text { [69] }\end{array}$ & 2014 & $\begin{array}{l}\text { Tracing of retinal blood vessels through edge } \\
\text { information }\end{array}$ & $\begin{array}{l}\text { DRIVE } \\
\text { ST ARE }\end{array}$ & - & - & $\begin{array}{l}0.9391 \\
0.9335 \\
\end{array}$ \\
\hline \multicolumn{7}{|c|}{ Multi Scale } \\
\hline Paper & Year & Title & Database & Sensitivity & Specificity & Accuracy \\
\hline $\begin{array}{l}\text { Martinez-Perez et } \\
\text { al. [45] }\end{array}$ & 1999 & $\begin{array}{l}\text { Retinal blood vessel segmentation by means of } \\
\text { scale-space analysis and region growing }\end{array}$ & DRIVE & 0.6389 & - & 0.9181 \\
\hline Vlacos et al. [46] & 2013 & $\begin{array}{l}\text { Multi-scale retinal vessel segmentation using line } \\
\text { tracking }\end{array}$ & DRIVE & 0.747 & 0.955 & 0.929 \\
\hline Tsai et al. [47] & 2013 & $\begin{array}{l}\text { Adaptive segmentation of vessels from coronary } \\
\text { angiograms using multi-scale filtering }\end{array}$ & - & - & - & 0.9566 \\
\hline Han et al. [48] & 2014 & Blood vessel segment ation in pathological retinal & DRIVE & 0.6770 & 0.9871 & 0.9473 \\
\hline & & image & ST ARE & 0.7043 & 0.9869 & 0.9573 \\
\hline
\end{tabular}

\section{References}

1. World Healt Organization, Definition, diagnosis and classification diabetes melitus and its complication, 1999.

2. S. Wild, G. Roglic, A. Green, R. Sicree, H. King, Global prevalence of diabetes estimates for the year 2000 and projection for 2030 , W o rld Health. 27 (2004) 1047-1053.

3. WHO, Definition and diagnosis of diabetes mellitus and intermediate hyperglycemia, in: Switzerland, 2006: p.50.

4. American Optometric Association, Diabetes eye care of the patient with diabetes mellitus, 2014.

5. S. You, E. Bas, D. Erdogmus, Principal curve based retinal vessel segmentation towards diagnosis of retinal diseases, IEEE Int. Conf. Healthc. Informatics Imaging Syst. Biol. 11 (2011) 331-337.
6. M.E. Martinez-Perez, A.D. Hughes, A. V. Stanton, S. a. Thom, N Chapman, A. a. Bharath, K.H. Parker, Retinal vascular tree morphology: A semi-automatic quantification, IEEE Tran s. Biomed. Eng. 49 (2002) 912-917.

7. C.G. Owen, A.R. Rudnicka, C.M. Nightingale, R. Mullen, S. a. Barman, N. Sattar, D.G. Cook, P.H. Whincup, Retinal arteriolartortuosity and cardiovascular risk factors in a multi-ethnic population study of 10 year-old children; The child heart and health study in England (CHASE), Arterioscler. Thromb. Vasc. Biol. 31 (2011) 1933-1938..

8. M. Preethi, R. Vanithamani, Review of retinal bloodvessel detection methods for automated diagnosis of Diabetic Retinopathy, Adv . Eng. Sci. (2012) 262-265. 
9. C.G. Owen, S.A. Barman, Bloodvessel segmentation methodologies in retinal images $-A$ survey, Comput. Methods Programs Biomed. 108 (2012)407-433..

10. N. Singh, L. Kaur, A survey on bloodvessel segmentation methods in retinal images, 2015 Int. Conf. Electron. Des. Comput. Networks Autom. Verif. (2015) 23-28.

11. S. Chhabra, B. Bhushan, Supervised pixel classification into arteries and veins of retinal images, Proc. Int. Conf. In nov. Appl. Comput. Intell. Power, Energy Control. with Their Impact Hum anit. CIPECH 2014. (2014) 59-62.

12. Y. Qian Zhao, X. Hong Wang, X. Fang Wang, F.Y. Shih, Retinal vessels segmentation based on level set and region growing, Pattern Recognit. 47 (2014) 2437-2446.

13. E.T.D. Ecencière, X.I.Z. Hang, G.U.Y.C. Azuguel, B.R.L. Aÿ, B. Éatrice, C. Ochener, C.A.T. Rone, P.H.G. Ain, J.O.H.N. Ichard, O.R. Arela, P.A.M. Assin, A.L.I.E. Rginay, B.É.C. Harton, J.E.A.N.L.K. Lein, Feedbackon a publicly distributed image database: the messidor database, (2014)231-234.

14. U.T.V. Nguyen, A. Bhuiyan, L. a. F. Park, K. Ramamohanarao, An effective retinal blood vessel segmentation method using multi-scale line detection, Pattern Recognit. 46(2013) 703-715.

15. D. Relan, T. Macgillivray, L. Ballerini, E. Trucco, Automatic retinal vessel classification using a least square-support vector machine in VAMPIRE*, (2014) 142-145.

16. M.M. Fraz, P. Remagnino, A. Hoppe, S. a. Barman, A. Rudnicka, C. Owen, P. Whincup, A model based approach for vessel caliber measurement in retinal images, 8 th Int. Conf. Signal Im age Technol. Internet Based Syst. SIT IS 2012r. (2012) 129-136.

17. A. Lisowska, R. Annunziata, G.K. Loh, D. Karl, E. Trucco, An experimental assessment of five indices of retinal vessel tortuosity with the RET-TORT public dataset, (2014) 5414-5417.

18. M.D. Saleh, C. Eswaran, A. Mueen, An automated blood vessel segmentation algorithm using histogram equalization and a utomatic threshold selection, J. Digit. Imaging. 24 (2011) 564-572.

19. M.B. Patwari, Classification and calculation of retinal blood vessels parameters, (n.d.) 1-7.

20. a. Elbalaoui, M. Fakir, K. Taifi, A. Merbouha, Automatic detection of blood vessel in retinal images, 201613 th Int. Conf. Co mput. Graph . Imaging Vis. (2016) 324-332.

21. A. Kumar, A.K. Gaur, M. Srivastava, A segment based te chnique for detecting exudate from retinal fundus image, Procedia Technol. 6 (2012) 1-9.

22. M.M. Fraz, S.A. Barman,P. Remagnino, An approach to lo calize the retinal bloodvessels using bit planes and centerline detection, Comput. Methods Programs Biomed. 108 (2012) 600-616.

23. L. Xu, S. Luo, A novel methodfor blood vessel detection from retinal images, Biomed. Eng. Online. 9 (2010) 14.

24. M. Foracchia, E. Grisan, A. Ruggeri, Luminosity and contrast normalization in retinal images, Med. Image Anal. 9 (2005) 14.

25. M. Vlachos, E. Dermatas, Multi-scale retinal vessel segmentation using line tracking, Comput. Med. Imaging Graph. 34 (2010) 213-227.

26. K.A. Goatman, A.D. Fleming, S. Philip, G.J. Williams, J.A. Olson, P.F. Sharp, Detection of new vessels on the optic disc using retinal photographs, Med. Imaging, IEEETrans. 30(2011)972-979.

27. Q. Li, J. You, D. Zhang, Vessel segmentation and width estimation in retinal images using multiscale production of matched filter responses, Expert Syst. Appl. 39(2012)7600-7610.

28. W. Azani, M.- Ieee, H. Yazid, S. Bin Yaacob, A Review : comparison between different type offiltering methods on the con trast variation retinal images, (2014) 28-30.
29. M.U. Akram, I. Jamal, A. Tariq, J. Imtiaz, Automated segmentation of blood vessels for detection of proliferative diabetic retinopathy, Biomed. Heal. Informatics. (2012) 232-235.

30. M.D. Saleh, C. Eswaran, An automated decision-support system for non-proliferative diabetic retinopathy disease based on MAs and HAs detection, Comput. Methods Programs Biomed. 108(2012) 186-196.

31. K. Sun, Z. Chen, S. Jiang, Y. Wang, Morphological multiscale enhancement, fuzzy filter and watershed for vascular tree extraction in angiogram, J. Med. Syst. 35(2011) 811-824.

32. L. Giancardo, F. Meriaudeau, T.P. Karnowski, Y. Li, K.W. Tobin, E. Chaum, Automatic retina exudates segmentation without a man ually labelled training set, Biomed. Imaging From Nano to Macro, 2011 IEEE Int. Symp. On. IEEE. (2011) 1396-1400.

33. M.U. Akram, A. Tariq, S. Nasir, S.A. Khan, Gabor Wavelet Based Vessel Segmentation in Retinallmages, IEEE. (2009).

34. M. Tamilarasi, K. Duraiswamy, Genetic based fuzzy seeded region growing segmentation for diabetic retinopathy images, Comput. Commun. Informatics Int. Conf. On. IEEE. (2013) 1-5.

35. B. Antal, A. Hajdu, An ensemble-based system for microaneurysm detection and diabetic retinopathy grading, Biomed. Eng. IEEETrans. 6 (2012) 1720-1726.

36. R. Bhattacharhjee, M. Chakraborty, Exudates, retinal and statistical features detection from diabetic retinopathy and normal fundus images: an automated comparative approach, Natl. Conf. Comput. Co mmun . Syst. Organ. by IEEE. (2012) 978.

37. S. You, E. Bas, D. Erdogmus, J. Kalpathy-Cramer, Principal curved based retinal vessel segmentation towards diagnosis of retinaldiseases, Proc. - 20111 st IEEEInt. Conf. Healthc. Informatics, Imaging Sy st . Biol. HISB 2011. (2011) 331-337.

38. G.S. Ramlugun, V.K. Nagarajan, C. Chakraborty, Small retinal vessel extraction towards proliferative diabetic retinopathyscreening, Expert Syst. with Appl. 39 (2012) 1141-1146.

39. B. Antal, I. Lazar, A. Hajdu, Z. Torok, A. Csutak, T. Peto, A Multi-level ensemble-based system for detecting microaneurysms in fundus images, 4th Int. Work. Soft Comput. Appl. IEEE. 10(2010) 137-142.

40. A.. Aibinu, M.. Iqbal, A.. Shafie, M.J.. Salami, M. Nilsson, Vascular intersection detection in retina fundus images using a new hybrid approach, Comput. Biol. Med. 40(2010)91-89.

41. K. Ram, Y. Babu, J. Sivanwamy, Curvature orientation histogram for detection and matching of vascular landmarks in retinal images, (2009).

42. S. Yacin, Retinopathy based on brightness variations in SDOCT retinal images, (2015).

43. E. Moghimirad, S. Hamid Rezatofighi, H. Soltanian-Zadeh, Retinal vessel segmentation using a multi-scale medialness function, Co m put. Biol. Med. 42 (2012) 50-60.

44. A.. Frangi, W.. Niessen, K.. Vincken, M.. Viergever, W. William, C. Alan, D. Scott, Multiscale vessel enhancement filtering, Med. Image Comput. Comput. Interv. MICCAI 98. (1998) 130.

45. M.E. Martinez-perez, A.D.Hughes, A.V. Stanton, S.A. Thom, A.A. Bharath, K.H. Parker, Retinal blood vessel segmentation by mean s of scale-space analysis and region growing, Proc. Second Int. Conf. Med. Image Comput. Comput. Interv. Springer-Verlag, London, UK, . (1999).

46. M. Vlacos, E. Dermatas, Multi-scale retinal vessel segmentation u sing line tracking, Comput. Med. Imaging Graph. (n.d.) 213-227.

47. Y.C. T sai, H.J. Lee, M.Y.C. Chen, Adaptive segmentation of vessels from coronary angiograms using multi-scalefiltering, Proc. - $2013 \mathrm{Int}$. Conf. Signal-Image Technol. Internet-Based Syst. SITIS 2013. (2013) 143-147.

48. Z. Han, Y. Yin, X. Meng, G. Yang, X. Yan, Blood vessel segmentation in pathological retinal image, (2014) 960-967. 
49. S. Chaudhuri, S. Chatterjee, N. Katz, M. Nelson, M. Goldbaum, Detection of blood vessels in retinal images using two dimensional matchedfilters, IEEETrans. Med. Imaging. 8 (1989) 263-269.

50. A.D. Hoover, V. Koznetsova, M. Goldbaum, Locating blood vessels in retinal images by piecewise threshold probing of a matched filter response, IEEET rans. Med. Imaging. 19 (2000) 203-210.

51. M. Al-Rawi, M. Qut aishat, M. Arrar, An improved matched filter for blood vessel detection of digital retinal images, Comput. Biol. Med. 37 (2007) 262-267.

52. M.. Cinsdikici, D. Aydin, Detection of blood vessels in ophthalmoscope images using MF/ant (matched filter/ant colony) algorith $m$, Co m put. Methods Programs Biomed. 96(2009) 85-95.

53. J. Odstrcilik, R. Kolar, T. Kubena, P. Cernosek, A. Budai, J. Hornegger, J. Gazarek, O. Svoboda, J. Jan, E. Angelopoulou, Retinal vessel segmentation by improved matched filtering: evaluation on a new high resolution fundus image database, IET Image Process. 7 (2013) $373-$ 383.

54. S. Fazli, a I. Transformations, A novel retinal vessel segmentation based on local adaptive histogram equalization, (2013)131-135.

55. Y. Xiang, X. Gao, B. Zou, C. Zhu, C. Qiu, X. Li, Segmentation of retinal blood vessels based on divergence and bot-hat Transform, (2014) 4-8.

56. R.B. Kawadiwale, Evaluation of algorithms for segmentation of retinal blood vessels, 00 (2015).

57. F. Farokhian, H. Demirel, Blood vessels detection and segmentation in retina using gabor filters, (2013) 104-108.

58. R. Masooomi, A. Mohtadizadeh, Retinal vessel segmentation using nonsubsam pled contourlet transform and multi-scale line detection, (2014).

59. F. Zana, J.C. Klein, A multimodal registration algorithm of eye fundu s images using vessels detection and hough transform, IEEE T rans. Med. Imaging 18. (1999) 419-428.
60. K. Sun, Z. Chen, S. Jiang, Y. Wang, Morphological multiscale enhancement, fuzzy filter and watershed for vascular tree extraction in angiogram, J. Med. Syst. (2010).

61. M.M. Fraz, S.A. Barman, P. Remagnino, A. Hoppe, A. Basit, B. Uyyanonvara, A. Rudnicka, C.G. Owen, An approach to localize the retinal blood vessels using bit planes and centerline detection, Comput. Methods Programs Biomed. (2011).

62. D. Singh, A new morphology based approach for blood vessel segmentation in retinal images, (2014).

63. B. Sindhu, J. Jeeva, Automated retinal vessel segmentation using morphological operation and threshold, Ijser.Org. 4 (2013) 1614-1617.

64. S. Wang, Y. Yin, G. Cao, B. Wei, Y. Zheng, G. Yang, Hierarchical retinal blood vessel segmentation based on feature and en semble learning, Neurocomputing. 149(2015) 708-717.

65. K. Saranya, B. Ramasubramanian, S. Kaja Mohideen, A novel approach for the detection of new vessels in the retinal images for screening diabetic retinopathy, 2012 Int. Conf. Commun. Signal Process. (2012) $57-61$.

66. S.W. Franklin, S.E. Rajan, Computerized screening of diabetic retinopathy employing blood vessel segmentation in retinal images, Biocybern. Biomed. Eng. 34 (2014) 117-124.

67. O. Chutatape, L.Z.L. Zheng, S.M. Krishnan, Retinal blood vessel detection and tracking by matched gaussian and kalman filters, P roc. 20th Annu. Int. Conf. IEEE Eng. Med. Biol. Soc. Vol.20 Biomed. En g. Towar. Year 2000 Beyond (Cat. No.98CH36286). 6 (1998) 44-49.

68. K.. Delibasis, A.. Kechriniotis, C. T sonos, N. Assimak is, A utomatic model-based tracing algorithm for vessel segmentation and diameter estimation, Comput. Methods Programs Biomed. 100. (2010) 108-122.

69. S. Khadijah, M. Zaki, M.A. Zulkifley, A. Nazari, Tracing of retinal blood vessels throughedge information, (2014) 28-30.

70. S.S. Patankar, A.R. Mone, J. V. Kulkarni, Gradient features and optimal thresholding for retinalblood vessel segmentation, 2013 IEEE Int. Conf. Comput. Intell. Comput. Res. IEEE ICCIC 2013 . (2013). 